\title{
Risk management for derivatives
}

\section{Frances Cowell}

138 Avenue Ledru Rollin, 75011 Paris, France.

Tel: + 330207809 6118, E-mail: frances.cowell@morleyfm.com

Received (in revised form): 16th October, 2006

Frances Cowell has worked in the investment management industry since 1983 starting as an equity analyst for Aetna Investment Management in Australia. Since then she has managed domestic and international equity and fixed interest portfolios, and asset allocation. In each case the focus has been applying quantitative techniques, with an emphasis on combining derivatives and physical investments, to construct and manage investment portfolios with predefined risk targets to meet specified investment objectives. In 1998, she moved to the UK to work for QUANTEC, a major provider of portfolio risk management systems, and in 2002 took up duties as Interim Head of Portfolio Risk at Morley Fund Management. She now works in the Portfolio Risk team, specialising in risk management for derivatives and hedge funds.

\section{Practical applications}

This paper

- describes how derivatives are used in portfolio management;

- explains how to measure the effective economic value of the investment achieved by a derivative transaction and the role of collateral;

- discusses the different types of risk associated with derivatives, the interaction between derivatives and conventional instruments and, from this,

- addresses the question of how derivatives risk can be measured, managed and, where necessary, controlled.

\section{Abstract}

For conjuring fear, few aspects of investments rival derivatives. This is not surprising given the history of august institutions humbled or even demolished as a result of poor risk management of their derivatives exposures. While some types of derivatives strategies certainly demand a very specific approach to risk management, it cannot be said that derivatives defy risk management. Derivatives Use, Trading \& Regulation (2006) 12, 228-243. doi:10.1057/palgrave.dutr.1850044

\section{INTRODUCTION}

The main reason that derivatives are perceived as being more risky than physical investment instruments is because they facilitate gearing and short selling. Gearing results from the ability to gain exposure to risky assets with a value much greater than the initial sum paid to effect the position. A sharp adverse market move can result in losses far exceeding the initial 'investment'. Short selling allows the investor to sell things he or she does not own, again with only a small initial payment, which can be dwarfed by the losses resulting from a sharp price appreciation. While these characteristics certainly can give rise to extreme outcomes, most derivatives held in investment portfolios (hedge funds are the notable exception) are managed in a way that
Derivatives Use, Trading \& Regulation, Vol. 12 No. 3, 2006, pp. 228-243 (C) 2006 Palgrave Macmillan Ltd $1357-0927 / 06 \$ 30.00$ 
avoids the risks of gearing and short selling. In fact, in many cases derivatives significantly reduce the risk of the overall portfolio.

\section{WHAT ARE THE OBJECTIVES OF INVESTING IN DERIVATIVES?}

Nearly all investment portfolios can benefit from using derivatives, either as a complement to conventional assets or as the basis for a separate investment product, such as a hedge fund or guaranteed return fund.

As a complement to conventional portfolios, derivatives are frequently used for liquidity management, tactical shifts, return enhancement and for risk management and control.

\section{Liquidity management}

Simple futures contracts, such as share price index futures and bond futures, can increase the efficiency of investing liquid assets that accumulate in portfolios as a result of dividend and other income flows, and new investment that arrives in small amounts. Rather than leaving these sums uninvested, futures contracts can be bought cheaply as temporary cover. Frictional or transactional liquidity thus forms collateral for bought futures contracts. When market conditions are favourable, or an economic volume of cash is accumulated, the futures can be sold and physical instruments bought in an Exchange-for-Physical transaction. Having negligible transactions costs, futures avoid the high costs of frequent trading of small volumes of physical instruments or the alternative of remaining underinvested in a rising market, risking underperformance. The use of futures in this way does not affect the risk of the portfolio (except that, by ensuring that the portfolio is fully invested, it lowers risk against the asset class benchmark). Return even can be enhanced if futures are bought and sold at prices that are advantageous relative to the underlying physical asset. This activity is scalable: the potential benefit of using futures to manage liquidity is virtually limitless due to the high volumes that can be transacted quickly on futures markets.

\section{Tactical shifts}

As with liquidity management, simple futures contracts can effect short-term asset allocation shifts with negligible transactions costs. Thus, a temporary shift from, say equities to bonds can be achieved by selling share price index futures and buying bond futures. As with the role of derivatives in liquidity management, futures contracts do not alter the risk of the portfolio, but can enhance return if bought below and sold above fair price, and by lowering transactions costs. For longer-term asset allocation shifts, asset swaps can be used in the same way as futures.

\section{Return enhancement}

Futures and options can be used to enhance returns to conventional portfolios, both in combination with liquidity management and as return enhancements in their own right.

- Futures in lieu of physical add risk-free return if they are bought at less than fair price, or are sold at greater than fair price. Fair price is easily calculated as a function of the price of the underlying asset, the interest rate and the time to expiry of the futures contract. This return enhancement is frequently achievable in the course of using futures for liquidity management or to effect tactical shifts.

- If the investor has some advantage in a particular asset class, such as a favourable tax position or better than average investment 
skill, then this can be 'transferred' to other asset classes. Physical securities are held in the favoured asset class, while futures are sold in that asset class and bought in another. For example, an investor may have particular skill in selecting European equities, but require some exposure to US stocks where his skill is only average. By combining bought physical European equities, selling futures on European equities and buying futures on US stocks, he can capture his European stockpicking skill while at the same time gaining a broad exposure to US stocks. This type of transaction is sometimes called a synthetic swap. The mechanism is the same as for tactical shifts: physical assets are held in the asset class where the manager has greatest advantage. At the same time, futures are used to achieve the desired portfolio asset allocation.

- Bought call options can enhance return in two ways:

- by providing a cost-efficient substitute for the underlying physical instrument; and

- by avoiding losses due to falls in the price of the underlying physical instrument.

- Bought put options can also enhance return in two ways:

- by benefiting from a fall in the price of the underlying asset; and

- by avoiding losses due to rises in the price of the underlying physical instrument.

- Sold options enhance return by attracting option premium, which the option seller retains if the options expire unexercised.

- Simultaneous bought and sold options can provide a cost-efficient substitute for the underlying physical instrument while protecting against unfavourable movements in the price of the underlying asset. For example, a bought call with an exercise price of $\$ 5.00$ combined with a sold call with an exercise price of $\$ 10.00$ gives exposure to share price movements in between. If the price of the underlying stock is $\$ 5.00$ or less at the time the options expire, then the investor loses the net premium paid for the options. If, on the other hand, the share price is $\$ 10.00$ or more when the options expire, the investor gains $\$ 5.00$ ( $\$ 10.00$ $\$ 5.00$ ) less the net premium paid.

- Buying and selling options according to their relative value (implied volatility), while ensuring that the overall position is marketneutral by means of delta-hedging, can be an important source of return enhancement that is independent of the return to the underlying physical investment. In other words, this source of return is unrelated to the direction of the securities on which the options are based. Because of its diversifying effect, it can significantly improve the riskreturn balance of the overall portfolio.

\section{Risk management and control}

Forwards, futures, swaps and options can all contribute to risk management and control of conventional portfolios. The simplest application is purchase and sale of foreign exchange forwards to hedge unwanted currency risk or the purchase of share price index or bond futures to manage liquidity, which protects the portfolio against being underinvested in a rising market. More sophisticated risk management is possible by carefully structured swap and option positions, which can allow the investor to target and hedge unwanted risk and retain or increase intended risk. The result is a portfolio that is finely tuned to its investment objectives and the views of the investment manager. 


\section{Investment products based on derivatives strategies}

Because they provide efficient means of hedging and managing risk and managing liquidity, futures, swaps and options can provide the basis for innovative investment products that meet specific risk-return objectives. For example:

- Smoothed return funds: Options, in conjunction with predefined decision rules, can help avoid the worst effects of sharp movements in physical asset prices and thus deliver portfolios with much reduced return volatility. For example, such portfolios can help meet demand for investments that participate in share market appreciation while avoiding the most brutal falls in value. Investors can choose the balance that suits them best between the level of protection against losses and participation in market growth. As many asset classes lack liquid options markets, many smoothed-return funds take advantage of the ability to use futures contracts to replicate the behaviour of options. Replicating options are discussed later in this article.

- Guaranteed minimum return funds: Actual options can deliver investments that will never fall below a specified value within a given timeframe, filling the need for even more risk averse investments. The benefit of using options to hedge these portfolios is that it avoids the need for expensive reserve funds. Investors can choose the balance that suits them best between the level of protection against losses and participation in market growth.

- Tactical overlay: These are specialist wholesale investment mandates designed to effect short-term modifications to the composition of an existing balanced portfolios. The overlay and underlying portfolios may be managed by different investment management companies. Futures and forwards are bought and sold to effect asset allocation shifts without transacting physical instruments. The main benefit is to reduce transactions costs, but overlay strategies can also enhance return in the same way that tactical shifts do.

- Protection overlay: They work in a similar way to tactical overlays but employ the option technology that is used for smoothed return or guaranteed minimum return funds, rather than simple bought and sold futures. The benefit is a cost-efficient hedge against unwanted volatility or unfavourable market movements without transacting physical instruments.

- Hedge funds: Futures, swaps and options are ideal for use in hedge funds as they facilitate both gearing and short selling. This allows the hedge fund to gain effective exposure to risky assets that is many times greater than the capital invested in the fund. They can also sell instruments short in anticipation of buying them back later at a lower price. Many hedge funds use futures, swaps and options to create offsetting long and short positions, giving a market-neutral portfolio that derives its return from the relative value of similar assets rather than the direction of the market itself.

\section{DERIVATIVES EXPOSURE: WHAT PROPORTION OF THE PORTFOLIO IS EFFECTIVELY EXPOSED VIA DERIVATIVES?}

For most applications of derivatives in conjunction with a conventional portfolio, the 
proportion of the portfolio that is invested using derivatives is quite small. The exceptions are investments based on derivatives where it is of course much more significant, and hedge funds, where the effective exposure through derivatives can greatly exceed the capital of the fund.

The first step in estimating the contribution of derivatives to the risk of a portfolio is to calculate the effective economic exposure to risky assets that is effected by the derivative position. This measure allows direct comparison of the derivative with the underlying physical instrument.

This calculation can be supplemented by calculations of capital employed and capital at risk, but neither of these is a substitute for the economic exposure. In addition, it is important to take into account the net, as opposed to the gross, economic exposure.

\section{CALCULATION OF ECONOMIC EXPOSURE}

For the purposes of risk analysis, the most appropriate method of calculating the effective exposure of a derivative contract is to use the face value calculation. This is simply the number of contracts held times the price of the contract times the point value of the contract. For example, the S\&P500 futures contract has a point value of $\$ 500$. An investor who has bought five contracts has an exposure to US equities of $\$ 2,500$ times the level of the index. So if the S\&P500 index is at 1,010, the exposure is $\$ 2,525,000$.

Calculation of the effective exposure facilitates a direct comparison of the risk of forwards, futures and swaps to the physical assets on which they are based. Thus, five bought S\&P500 future contracts, backed by $\$ 2,525,000$ of liquid collateral is economically identical to holding $\$ 2,525,000$ in US equities.

\section{CAPITAL EMPLOYED}

The accounting treatment of futures differs from the economic exposure, and this can be a source of confusion. It is based on the amount of capital actually transferred in relation to the derivatives position. This reflects initial and any variation margins, which in turn may reflect unrealised losses. This measure is intended to track cash flows, but otherwise gives no representation of economic exposure. Table 1 illustrates the differences.

The example shows a portfolio with a total value of $\$ 100,000,000$ which is invested in share price index futures and cash. Two hundred futures contracts were bought at 2743.4 and the current price is 2750.0 . The point value of the contract is $\$ 100$ and the initial margin per contract is $\$ 3,000$. The capital employed is equal to the initial margin paid plus any unrealised profit and loss, in this case $200 \times \$ 3,000+$ $\$ 132,000(200 \times \$ 100 \times(2750.0-2743.4))$, or $\$ 732,000$. The economic exposure on the other hand is $200 \times \$ 100 \times 2750.0$, or $\$ 55,000,000$. A one per cent increase in equity prices will result in a further profit of $\$ 550,000$ $(200 \times \$ 100 \times 27.50)$, or one per cent of the economic exposure. By contrast, the same price increase would represent a 75 per cent increase on the capital employed!

\section{CAPITAL AT RISK}

This measure is sometimes applied to bought options, and reflects the fact that these positions have a known maximum loss, equal to the option premium paid. While this information is important for the purposes of some types of risk 
Table 1: Economic exposure versus accounting treatment for futures

$\begin{array}{lll}\text { Point value of contract: } & \$ 100 & \\ \text { Initial margin per contract } & \$ 3,000 & \\ \text { Number of contracts bought } & 200 & \\ \text { Number of contracts sold } & 0 & \\ \text { Price at which futures traded } & 2743.4 & \\ \text { Current futures price } & 2750.0 & \\ \text { Initial cash position } & \$ 100,000,000 & \text { Capital employed } \\ & & \$ 600,000 \\ \text { The valuation statement } & \text { Economic exposure } & \$ 132,000 \\ \text { Initial margin } & \$ 600,000 & \$ 0 \\ \text { Unrealised profit and loss } & \$ 132,000 & \$ 732,000 \\ \text { Cash collateral } & \$ 54,268,000 & \$ 99,268,000 \\ \text { Equity futures } & \$ 55,000,000 & \$ 100,000,000 \\ \text { Cash } & \$ 45,000,000 & \\ \text { Total } & \$ 100,000,000 & \end{array}$

analysis, such as worst loss analysis, it can give a misleading impression because it ignores the participation of the option to favourable price changes in the underlying instrument.

\section{GROSS VERSUS NET}

Portfolios often combine bought and sold positions in the same, or very similar derivatives contracts. Clearly a bought future in one delivery month that is offset by an equivalent sold future in another delivery month is less risky than either the bought or sold positions by themselves. For this reason there must be some provision for calculating the net exposure so that enough collateral is allocated to ensure against gearing, but not too much that other desirable transactions are impeded. For example, if an investor buys five S\&P500 futures contracts and the price stands at 1020.0, the exposure is equivalent to $\$ 2,550,000$ worth of US equities.
If this position is augmented with three sold contracts (equivalent to $\$ 1,530,000$ in US equities), then the exposure of the position is reduced to $\$ 1,020,000$.

\section{REQUIRED RISK CONTROLS}

Having determined what proportion of the portfolio is exposed using derivatives, the task of risk management falls into two main categories: operational risk and investment risk.

\section{OPERATIONAL RISK}

This category deals with the risk of errors in implementation and bookkeeping, it includes:

- Instrument risk: This is the risk that derivatives will be used inappropriately. The most common error is incorrect attribution of liquid collateral, followed by errors in valuation and the danger of selling call 
options for which insufficient underlying securities are held to ensure delivery. These risks are easily eliminated with the assistance of appropriate management systems and procedures.

- Collateral management: As mentioned above, ensuring correct management of liquid collateral is the most common and basic risk control measure. There is always the temptation to invest collateral in risky assets, which if not well managed, can compound the risk of the derivative.

- Counterparty risk: Over-the-counter derivatives, such as forwards, swaps and bought options are subject to the risk that the investor or intermediary with whom the agreement is struck is unable to honour the contract. This risk is managed in the same way as counterparty or credit risk for fixed interest securities in conventional portfolios and treasury operations: that is, by setting and enforcing limits on the face value exposure to any nominated counterparty or intermediary. While counterparty risk is integral to the risk of the portfolio, it is effectively a type of credit risk, and so is usually managed as part of the cash or money market portfolio, separate from the risk management of the derivatives themselves.

Operational risk measures are necessary but not sufficient. They usually can be managed using automated checking systems and because they deal chiefly with breaches of mandates and statutory or fiduciary obligations, are best eliminated entirely.

\section{INVESTMENT RISK}

The second category of risk management seeks to quantify the contribution of derivative positions to the investment objectives of the portfolio: for example, by quantifying how much they add to or reduce the return and risk of the portfolio and if they introduce any other, unwanted or contradictory risks. At this level, risk management, to be effective, must take into account the objectives of the portfolio and the reasons for including derivatives. It therefore calls for much more judgment than does operational risk.

Active returns cannot be achieved without taking risks, so quantifying investment risk is crucial to understanding how the portfolio will achieve its objectives. If the risk is too low, for example, there is little chance of the portfolio earning the required returns. On the other hand, if the risk is too high, or drawn from sources that are unlikely to deliver positive returns, then the portfolio will be subject to high and inappropriate risks for which there are unlikely to be compensating active returns.

An often-overlooked aspect of derivatives is basis risk. This is the risk that the future or option contract is not a perfect hedge for the underlying assets. For example, the S\&P500 futures contract is usually only an approximation for the portfolio with which it is used. Basis risk is more noticeable for exchange-traded contracts, as over-the-counter contracts can generally be tailored to more closely suit the underlying portfolio of physical assets.

Once the question of collateral has been dealt with, many portfolios can be subject to risk analysis in much the same way as if the portfolio consisted only of conventional assets. Forwards, futures and swaps are substituted by the appropriate physical assets. For example, for equities or balanced portfolios the expected tracking error and sources of tracking error are computed using a risk factor model to construct 
a risk profile. A bond portfolio would have duration, convexity calculated as well as perhaps some measure of Value at Risk (VaR) and perhaps some stress testing or scenario analysis.

For return enhancement, return smoothing and risk control using options, a more complex approach is required. The distinguishing feature of options is that they allow the investor to participate in either upward or downward shifts in market prices, effectively insuring against the effects of adverse directional shifts. This asymmetry of returns causes problems when risk factor models are applied to portfolios with options in them, so another approach must be tried.

The potential solutions fall into two main categories:

1 Maintain the risk factor approach used for conventional portfolios with modifications to accommodate options.

2 Apply risk measures that do not rely on normal-like return distributions.

\section{CONVENTIONAL RISK FACTOR ANALYSIS WITH MODIFICATIONS FOR OPTIONS}

This relies on the fact that, for small changes in the price of the underlying asset, an option behaves in a predictable way relative to that asset. The relationship between the two is the option's delta. This is a number between -1.0 and 1.0 that measures the change in the price of the option for a given change in the underlying physical. Thus, an option with a delta of 0.25 will go up $\$ 0.25$ if the underlying stock goes up $\$ 1.00$.

For equity and balanced portfolios with relatively modest option components, such as those used for return enhancement or small tactical shifts, simply including the option as the delta-weighted stock equivalent will give acceptable results. It is important to remember that the option delta holds only for small changes in the underlying asset, and over short time horizons. So the results given are not directly comparable to a portfolio without options, if the percentage of the portfolio invested using options is small, the portfolio-level effect will be unimportant. On the other hand, the more options there are in the portfolio, the more necessary is some further analysis.

Knowing that option deltas are not stable, it makes sense to measure the stability of the delta. This is given by the option's gamma, which is the change in delta for a small change in the price of the underlying asset. A positive gamma indicates that the portfolio stands to benefit from volatility in the underlying market, a negative gamma indicates that, other things being equal, the portfolio is likely to suffer (compared to the equivalent physical position) in volatile times.

\section{RISK MEASURES THAT DO NOT RELY ON NORMALLY DISTRIBUTED RETURNS}

For portfolios, such as smoothed-return portfolios, guaranteed return portfolios and hedge funds that comprise significantly or even uniquely of options, a simple delta-modified risk factor approach is unsatisfactory in two respects:

1 The delta and gamma at the portfolio level may be too unstable to give meaningful results.

2 Factor-based analyses do not accommodate the asymmetric return distributions that characterise these types of portfolio. 
3 Being based on medium and long-term historical data, the results they give may have limited relevance to portfolios, such as most hedge funds, that have high rates of turnover.

Such portfolios require an approach that specifically addresses the issue of asymmetry of returns. There are three main measures:

1 Value at Risk;

2 Scenario analysis and stress testing;

3 Sensitivity analysis.

\section{Value at Risk (VaR)}

$\mathrm{VaR}$ is similar to tracking error in that it quantifies a return that corresponds to a given probability. The main difference is that, whereas tracking error gives the magnitude of the standard deviation from the benchmark, which is equally likely to be positive or negative, $\mathrm{VaR}$ focuses on downside risk. For this reason, it is frequently used for portfolios with asymmetric return distributions, or where extreme outcomes, often called 'fat tails' ${ }^{1}$ are more likely than would be predicted by a normal distribution. It was developed originally to measure the overnight risk in bank treasury portfolios comprising mostly interest rate instruments, and comes in three varieties.

1 Historical VaR: Measures the return of the portfolio at frequent intervals, such as daily, and computes the mean and standard deviation of those returns. From this it estimates the size of the likely loss for a given probability, such as one in 20 , usually referred to as the 95 per cent VaR. It is important to note that, while the calculation accommodates asymmetry in individual instrument returns, it still imposes the assumption of normality at the portfolio level.
2 Non-parametric VaR: Works in a similar way to Historical VaR, but allows for nonnormality of returns for the portfolio itself as well as for individual positions.

3 Monte Carlo VaR: From the statistics obtained from historical returns, a large number of future returns are simulated, from which the size of loss for a given probability can be inferred. Monte Carlo VaR can accommodate asymmetry and fat tails at the instrument as well as the portfolio level. This can be very helpful, for example a 95 per cent VaR combined with a 99 per cent VaR can disclose important exposures to extreme events that are not evident from the 95 per cent measure by itself.

One of the main advantages of $\mathrm{VaR}$ is that, because it gives a single number, it can be used as a summary statistic for frequent risk measurements, such as daily. This is particularly useful for hedge funds that often have very high turnover where frequent in-depth analysis is neither practicable nor helpful. VaR has five limitations:

1 It was designed initially to describe the overnight risk taken by banks when managing their treasury portfolios, and so must be handled with care when applied to any investment portfolio that is intended to be held longer. While positions in hedge funds tend to be held for shorter periods than conventional investment portfolios, they still typically are held for between a week and a month, subjecting them to risk on a different scale than treasury overnight positions.

2 There is as yet no standard methodology for calculating these risk measures (although 95 
per cent, 5-day $\mathrm{VaR}$ is increasingly popular for some types of hedge funds), so a VaR computed by one investment manager may not compare directly with that prepared by another.

3 Most measures of $\mathrm{VaR}$ are derived, either directly or indirectly, from past returns, so implying that future patterns of return will be similar - which may not be the case.

4 Because it concentrates on downside risk, $\mathrm{VaR}$ ignores the link between risk and positive returns. In other words, it does not say anything about how portfolio risk will contribute to investment objectives. This concentration on negative returns is especially important when the overall distribution of returns is asymmetrical.

$\mathrm{VaR}$ is very useful as a supplement to other risk measures, but is unsatisfactory by itself. It is therefore usually combined with one or more of the following:

- Scenario analysis is often used in conjunction with $\mathrm{VaR}$. As the names suggest, scenario analyses subject the portfolio to predefined scenarios in order to estimate their effect on portfolio return. The advantages are that they give a portfolio-wide measure of risk, accommodate all instruments and strategies and do not impose any direct assumptions about the distribution of returns, either for individual assets or for the portfolio overall. They also accommodate favourable as well as unfavourable outcomes, thereby giving an important perspective to the portfolio's risk. Their main disadvantage is that if the scenario or stress test is poorly defined, then the results will be misleading. Scenario analysis, despite being conceptually simple, is in fact very complex to implement. To see why, consider a simple, eight-asset portfolio to which five scenarios are applied. The investor must forecast returns for each asset class and each scenario, in other words 40 forecasts. But these return forecasts must be consistent within each scenario, so implying forecasts of correlations. For eight assets there are 28 pairs, so 140 correlations for all five scenarios. To complete, the analysis requires probabilities for each scenario. This amounts to 184 forecasts. Since most balanced funds have many more than eight asset classes, the total is usually much more. The likelihood of error is related to the number of forecasts, so scenario analysis leaves plenty of opportunity for mistakes. While still potentially very helpful, the results it gives should be handled with care.

- Stress testing is a type of scenario analysis, applying only one scenario at a time, which usually involves defining an economic shock and working out what impact it would have on the portfolio. Most such shocks mimic some historical event, such as October 1987 or the 1997 Asian crisis. This type of analysis has obvious appeal, as investors worry about what would happen if these events were to recur. The problem with this is that such events rarely repeat themselves: the reason they are shocks is that they are unique and unforeseen. For example, the information derived from the two events referred to above gives little insight into the consequences of the Russian debt default.

- Sensitivity analysis computes the impact on a portfolio of small changes in the values of assets and factors to which it is exposed. For example, a portfolio comprising equities and bonds will be subject to various 
combinations of small changes in equity prices and interest rates, yield curve shifts, exchange rates and so on. The main advantages of this measure are its conceptual simplicity and the fact that there is no need to define meaningful scenarios or shocks. Its limitation is that, having been designed for short-term portfolios, it does not measure risks that affect medium and long-term returns, or the impact of large changes in asset prices that might be associated with an economic shock.

Because none of these measures by themselves give sufficient insight into the risk exposures of a complex portfolio, they tend to be employed in some combination, for example, 95 and 99 per cent VaR might be combined with stress tests that apply two or three disaster scenarios to the portfolio.

\section{RISK MANAGEMENT VERSUS RISK CONTROL}

When derivatives are used principally to control portfolio risk, the most interesting question is whether or not they are working.

Risk reduction strategies include simple transactions such as using forwards to eliminate currency risk, stock index or bond futures to gain exposure for uninvested cash, and more complex transactions using options to reduce or eliminate the risk of negative returns. The simplest way to find out if the derivatives are doing their risk-reduction job is to measure the risk of the portfolio (using any standard risk measurement tool) both with and without the derivatives. If the derivatives are doing their job, then the portfolio will be less risky with them than without. If the risk tool allows it, risk profiling should show that the risk reduction has been targeted appropriately.

This comparative approach is very effective for quantifying the effect of basis risk. For portfolios that use forwards, futures and swaps, but not options, the use of standard risk tools without modification is generally acceptable. For quantifying the effectiveness of risk reduction, there is an enormous advantage in applying a conventional risk factor approach, as this permits quantifying the sources of risk as well as overall risk. For example, if foreign exchange forward contracts are used to hedge currency risk, then the risk factor model should show a reduction of overall risk and the appropriate reduction of the currency risk that is hedged.

Many portfolio strategies rely on options or option-like strategies to deliver minimum return portfolios or smoothed-return portfolios, also called portfolio protection. Where the protection or smoothed returns are achieved by means of bought options, then any VaR or stress test should show if it is working or not. Often, however, the protection or smoothing is implemented using replicating options, also known as a dynamic hedge. The main risk of dynamic hedges is that the cost is not knowable until the hedge is removed or has expired. The other risk is that, if poorly implemented, or if market conditions differ significantly from those anticipated, the hedge may not be as effective as expected, or may cost more or both.

Portfolio protection relies on option theory. The most common applications use some variant of Black-Scholes option pricing technology (see Box 1). Portfolio protection can take the form of an option purchased on market or over-thecounter, or the investment manager can create the required option. An option thus created is often referred to as a replicating option. It uses a 
carefully defined mix of a risky asset (or basket of risky assets) to be protected and the risk-free asset (or cash). The basket of risky assets in this mix is structured to resemble as closely as possible the asset allocation of the portfolio to be protected. It can consist of physical assets, but in practice is usually made up of futures, which significantly reduces transactions costs and allows short sales, which are required to replicate bought put options. Using futures contracts in lieu of physical assets can introduce basis risk but this is usually preferable to incurring the high costs of transactions of physical assets.

The proportion of risky and riskless assets in the replicating option is determined by the delta of the option being replicated. When the value of the underlying investment is the same as the value at which it is protected, the delta is about 0.5 . In this case, for a replicating call the portfolio has 50 per cent in risky assets and 50 per cent in cash. For a replicating put, the portfolio sells 50 per cent of its face value in risky assets and holds 150 per cent in cash. As the value of the option increases, so does the delta, until the portfolio is 100 per cent invested in risky assets (for a call) and has sold 100 per cent of its face value in risky assets (for a put). As the value of the option decreases, the delta does too, until the replicating option comprises 100 per cent cash. In either case, the investor is buying in a rising market and selling in a falling market.

It is called a dynamic hedge because the delta, and therefore the mix of risky and riskless assets in the replicating portfolio changes constantly.

The main difference between an option bought on market or over-the-counter and a replicated one is that the cost of the bought option is known at the outset, while the cost of the replicating option can only be known with certainty at the expiry of the option or protection period. To protect a physical portfolio with actual or replicating put options, the investor may choose to pay the manager a fixed fee at the start of the option period, in which case the manager bears the risk that the actual cost of replicating the option turns out to be higher. Otherwise the investor may choose to pay the ongoing cost of the replicating option. Paying the investment manager a fixed fee for protection has the advantage that the investor can ask a number of managers to quote prices for the protection, and choose the most attractive one. Professional protection managers have a significant cost advantage over individual investors, because they can aggregate the hedge programmes of different portfolios. This can materially reduce both the cost and risk of replicating options, an advantage that is hard to overstate, particularly in volatile markets.

Evaluating the effectiveness of portfolio protection is conceptually no different from other derivative-based risk management strategies. If protection is effected mainly using options, then some combination of VaR, stress testing and delta-weighted factor analysis is appropriate. If futures are used to replicate options, then both these and risk factor analysis can be employed. To test if the risk management is working, the risk of the portfolio is measured with and without the derivatives positions. The portfolio should be less risky with than without the derivatives. Risk factor analysis should show that this risk reduction is spread evenly across the portfolio.

\section{SUMMARY}

Derivatives are used increasingly in investment management because of their liquidity, cost- 
effectiveness and flexibility. They are used both in conjunction with conventional, physical assets and as the basis for structured investment products. Common uses of derivatives include:

- liquidity management, also known as efficient portfolio management;

- tactical shifts between asset classes or individual securities;

- return enhancement;

- risk management and control, including hedging of currency risk.

Structured investment products usually fall into one of the following categories:

- hedge funds;

— smoothed return funds;

- guaranteed minimum return funds;

- tactical overlay funds;

- protection overlay funds.

Whether derivatives are used for efficient portfolio management, return enhancement or risk control, it is important to quantify accurately the economic exposure achieved by derivatives, and to distinguish between net and gross exposure.

The approach to risk measurement and management should reflect the objectives of using derivatives in the first place: for efficient portfolio management, the risk of the portfolio should not be increased by the use of derivatives. For return enhancement, naturally one would expect to see some increase in risk. On the other hand, if derivatives are deployed for the purpose of risk control, the portfolio risk profile should show a reduction of risk overall, and in particular in the sources of risk being controlled.
From the point of view of risk estimation and profiling, forwards, futures and swaps are economically no different from physical assets, and can be analysed in the same way using risk tools developed for those assets. Options and option-like instruments, such as swaptions, are economically different from other instruments because they have asymmetric return distributions. Conventional risk management can be applied to portfolios with options in them, but the resulting analysis lacks robustness, being valid only for very small changes in market prices and at a particular point in time. A more robust analysis that measures the portfolio's susceptibility to market volatility is given by VaR but this analyses does not facilitate the detailed risk decomposition that is available for conventional assets.

Scenario analysis, stress testing and sensitivity analysis can provide some insights, but suffer other limitations. Scenario analysis and stress testing depend on defining meaningful scenarios and shocks, while sensitivity analysis tend to work only for small changes in asset prices and short time horizons.

Risk analysis for return enhancements and hedge funds is complicated by high turnover. Risk analysis based on a snapshot loses validity very quickly as the composition of the portfolio changes. In these cases, the snapshot approach should be complemented by some summary risk measure, such as tracking error or $\mathrm{VaR}$ measured at frequent intervals, such as daily.

One approach to evaluating risk for efficient portfolio management and risk control is to conduct a portfolio-wide risk analysis using the risk tools appropriate to the instruments in the portfolio, and apply this to the portfolio with and without the derivatives. The difference in 
the analysis will illustrate how much the risk of the portfolio is altered by the derivatives positions, and if detailed risk profiling is conducted, which sources of risk have been modified.

As with any approach to risk management, the effectiveness of the measures lies in understanding the limitations and strengths of each. Usually this implies applying multiple risk measures to gain a balanced view of the portfolio with and without derivatives. Whichever approach is adopted, it is imperative to avoid treating the analysis as a black box, and to remember that the results are only as good as the information used and the assumptions implicit in the analysis.

\section{Box 1: Option pricing theory and replicating options}

To calculate the price of an option and to estimate the delta for the replicated option, the following information is required:

- the value of the underlying investment;

- the value at which the option takes effect, otherwise known as the exercise price;

- the time to expiry of the option;

- the risk-free interest rate;

- the volatility, or riskiness of the underlying investment.

The formula that combines this information to give the price of the option incorporates a number of assumptions about markets that, when violated, can change the effective option cost. The first of these is that both the interest rate and portfolio volatility will not change during the life of the option. Although these assumptions are frequently violated, most replicating options are robust enough to endure all but the most extreme departures from theory. More important are the assumptions of zero transactions costs and continuous asset price movements. Because most replicating options undertake a large number of transactions, paying transactions costs can significantly add to the cost of protection. And because asset prices, and therefore portfolio values, sometimes change abruptly, rather than in a smooth series of infinitely small increments, the actual cost of replicating an option can be much higher than the cost predicted by theory. Differences of ten per cent between closing prices, and opening prices the following day are not uncommon in some markets. Gapping, or jump risk can cause the actual cost of the replicating strategy to significantly exceed the estimated cost. While theory requires that the investor adjust the replicating portfolio simultaneously with changes in value of the underlying portfolio, in reality the investor always lags the market, which usually means buying at a higher price and selling at a lower price than is predicted by the theory. This effect is demonstrated in the next example. 


\begin{tabular}{|c|c|c|c|}
\hline \multicolumn{4}{|c|}{ Example: Actual versus replicating options } \\
\hline Market data & At start & $\begin{array}{l}\text { After market } \\
\text { move of } 10 \%\end{array}$ & $\begin{array}{l}\text { After market } \\
\text { move of }-10 \%\end{array}$ \\
\hline Length of period in days & 153 & 153 & 153 \\
\hline Initial value of equities held & $\$ 100,000,000$ & $\$ 100,000,000$ & $\$ 100,000,000$ \\
\hline Short-term interest rates & $7.5 \%$ & $7.5 \%$ & $7.5 \%$ \\
\hline Dividend yield & $1.5 \%$ & $1.5 \%$ & $1.5 \%$ \\
\hline S\&P500 physical & $1,328.7$ & $1,461.6$ & $1,315.4$ \\
\hline S\&P500 futures & $1,362.1$ & $1,498.4$ & $1,348.5$ \\
\hline Point value of futures & $\$ 500$ & $\$ 500$ & $\$ 500$ \\
\hline Option exercise price & 1,475 & 1,475 & 1,475 \\
\hline Estimated volatility & $25 \%$ & $25 \%$ & $25 \%$ \\
\hline Call option price & 79.55 & 178.90 & 83.71 \\
\hline Call option delta & 0.51 & 0.77 & 0.53 \\
\hline \multicolumn{4}{|l|}{ Bought call option } \\
\hline Number of call options purchased & 151 & & \\
\hline Face value of options & $\$ 100,318,360$ & & \\
\hline Option premium paid & $\$ 6,005,663$ & & \\
\hline Cash collateral held & $\$ 100,000,000$ & $\$ 100,000,000$ & $\$ 100,000,000$ \\
\hline Value of call option & $\$ 6,005,663$ & $\$ 13,506,715$ & $\$ 06,320,426$ \\
\hline Cost of option & $\$ 6,005,663$ & $\$ 6,005,663$ & $\$ 6,005,663$ \\
\hline Value of portfolio & $\$ 100,000,000$ & $\$ 107,501,052$ & $\$ 100,314,763$ \\
\hline \multicolumn{4}{|l|}{ Replicating call option } \\
\hline Number of futures purchased & 77 & & \\
\hline Face value of portfolio protected & $\$ 100,478,332$ & & \\
\hline Cash collateral held & $\$ 100,000,000$ & $\$ 100,000,000$ & $\$ 100,000,000$ \\
\hline Unrealized value of futures & - & $\$ 5,115,572$ & $-\$ 511,557$ \\
\hline Value of portfolio & $\$ 100,000,000$ & $\$ 105,115,572$ & $\$ 99,488,443$ \\
\hline
\end{tabular}




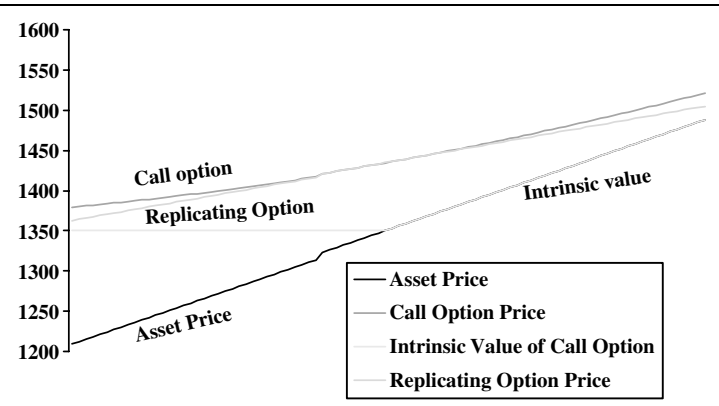

In the case of both a rise and a fall of 10 per cent, the replicating option performs worse than the actual option. The graph shows why. The curved line describes the actual option, while the straight line at its tangent describes the replicating portfolio, with the slope of the line equivalent to the option's delta. When the market goes up, the actual option curves up while the replicating option moves along the straight line, exposing a gap between their values. As the market falls, the curvature of the actual option gives it the advantage over the replicating option. In practice, the mix of risky and riskless assets in the replicating option is adjusted to match the delta of the actual option as it changes. The more frequently this rebalancing occurs, the closer in theory will be the behaviour of the replicating to the actual option. In practice, the costs of rebalancing add to the cost of the replicating option relative to the actual.

\section{Note}

1 Fat tails are blamed for the relatively frequent occurrences of events described as 'one in one hundred years'. The 'fatness' of the 'tail' represents the increased likelihood of these events over that predicted by the normal distribution. 